\title{
Acidic fibroblast growth factor attenuates type 2 diabetes-induced demyelination via suppressing oxidative stress damage
}

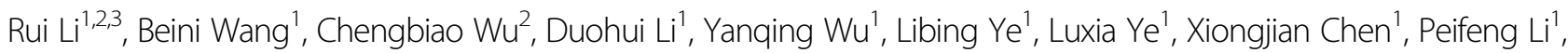 \\ Yuan Yuan', Hongyu Zhang ${ }^{1}$, Ling Xie', Xiaokun $\mathrm{Li}^{1}$, Jian Xiao (1) ${ }^{1}$ and Jian Wang ${ }^{1}$
}

\begin{abstract}
Prolonged type 2 diabetes mellitus (T2DM) produces a common complication, peripheral neuropathy, which is accompanied by nerve fiber disorder, axon atrophy, and demyelination. Growing evidence has characterized the beneficial effects of acidic fibroblast growth factor (aFGF) and shown that it relieves hyperglycemia, increases insulin sensitivity, and ameliorates neuropathic impairment. However, there is scarce evidence on the role of aFGF on remodeling of aberrant myelin under hyperglycemia condition. Presently, we observed that the expression of aFGF was rapidly decreased in a db/db T2DM mouse model. Administration of exogenous aFGF was sufficient to block acute demyelination and nerve fiber disorganization. Furthermore, this strong anti-demyelinating effect was most likely dominated by an aFGF-mediated increase of Schwann cell (SC) proliferation and migration as well as suppression of its apoptosis. Mechanistically, the beneficial biological effects of aFGF on SC behavior and abnormal myelin morphology were likely due to the inhibition of hyperglycemia-induced oxidative stress activation, which was most likely activated by kelch-like ECH-associated protein 1 (Keap1)/nuclear factor erythroid-derived-like 2 (Nrf2) signaling. Thus, this evidence indicates that aFGF is a promising protective agent for relieving myelin pathology through countering oxidative stress signaling cascades under diabetic conditions.
\end{abstract}

\section{Introduction}

Type 2 diabetes mellitus (T2DM), a clinical chronic disease worldwide, has become an epidemic disease accompanying insulin resistance and insulin secretion defect $^{1,2}$. Statistically, the annual expenditure for T2DM management is approximately $\$ 825$ billion around the world every year, and T2DM produces an enormous financial burden for the health care system $^{3,4}$.

Correspondence: Jian Xiao (xfxj2000@126.com) or

Jian Wang (jianwang0516@126.com)

${ }^{1}$ Department of Hand Surgery and Peripheral Neurosurgery, The First Affiliated Hospital and School of Pharmaceutical Sciences, Wenzhou Medical University, 325000 Wenzhou, Zhejiang, China

${ }^{2}$ Research Center, Affiliated Xiangshang Hospital, Wenzhou Medical University, 315700 Ningbo, Zhejiang, China

Full list of author information is available at the end of the article

These authors contributed equally: Rui Li, Beini Wang, Chengbiao Wu,

Duohui Li

Edited by M. Agostini
Unfortunately, there are no effective therapeutic strategies to cure this chronic disease, except for rigorous glucose control ${ }^{5}$. Long-term hyperglycemia in the peripheral nervous system (PNS) causes nerve damage and cytotoxicity, which leads to myelin sheath vacuolar degeneration and the generation of numerous onion bulb-shaped formations. If these pathological changes continue throughout T2DM progression, the impaired nerve fibers undergo segmental demyelination, eventually leading to diabetic peripheral neuropathy (DPN). Experimental and clinical studies have demonstrated that patients with demyelinating neuropathy have a higher risk of developing sensory deficits and muscle weakness, as well as foot ulceration ${ }^{6-9}$. Thus, preventing demyelination progression in T2DM is a critical issue for ameliorating abnormal nerve function.

\section{(c) The Author(s) 2021}

(c) (i) Open Access This article is licensed under a Creative Commons Attribution 4.0 International License, which permits use, sharing, adaptation, distribution and reproduction c. in any medium or format, as long as you give appropriate credit to the original author(s) and the source, provide a link to the Creative Commons license, and indicate if changes were made. The images or other third party material in this article are included in the article's Creative Commons license, unless indicated otherwise in a credit line to the material. If material is not included in the article's Creative Commons license and your intended use is not permitted by statutory regulation or exceeds the permitted use, you will need to obtain permission directly from the copyright holder. To view a copy of this license, visit http://creativecommons.org/licenses/by/4.0/. 
As supporting gliocytes in the PNS, Schwann cells (SCs) produce consecutive layers of the plasma membrane that enwrap axons; these layers form myelin sheaths as they mature. During the process of remyelination, SCs are pivotal for regulating myelin thickness, maintaining the normal conduction of electrical impulses and providing trophic support for axon regeneration ${ }^{10,11}$. Emerging evidence suggests that $\mathrm{SCs}$ are crucial to modulate the pathogenesis of DPN and chronic hyperglycemia can induce SC dysfunction in diabetic humans and felines ${ }^{12-15}$. Given the pivotal role of SCs in myelin maintenance and nerve regeneration, enhancing the proliferation and migration of SCs and restraining their apoptosis may be an ideal therapy to attenuate demyelination in acute or chronic diabetes.

Acidic fibroblast growth factor (aFGF), also known as FGF1, is an autocrine/paracrine regulator that controls cell proliferation, migration, and survival through binding its high-affinity receptors (FGFR1-4) ${ }^{16}$. The application of recombinant human aFGF to facilitate wound or burn repair in diabetic patients has achieved excellent clinical therapeutic effect. Furthermore, previous investigations confirmed that aFGF is expressed extensively in the mammalian nervous system and the progressive decrease of aFGF is identified in the peripheral blood of patients with $\mathrm{T}_{2} \mathrm{DM}^{17,18}$. aFGF is also shown to have powerful neuroprotective and neuroregenerative effects that facilitated neurite outgrowth, stimulated motor and sensory axon regeneration, and prevented neuronal death postaxotomy ${ }^{19-21}$. Importantly, aFGF has emerged as a promising solution to relieve diabetic hyperglycemia and enhance insulin sensitization ${ }^{22,23}$. Conversely, this antidiabetic effect was abolished when the aFGF gene was knocked out ${ }^{24,25}$. Based on the important role of aFGF in regulating glucose uptake, we speculate that aFGF protects SCs from high-glucose-induced excessive apoptosis, ultimately contributing to myelin regeneration and remodeling after pathological changes to the peripheral nerve under diabetic condition. However, we still do not understand the mechanism underlying aFGF ameliorates demyelination.

Chronic hyperglycemia in diabetes triggers various pathological changes, including advanced glycation, polyol pathway activity, and superoxide-induced free radical formation ${ }^{26}$. Recently, a growing body of studies has suggested that oxidative stress is a major hallmark of T2DM and its associated complications ${ }^{27,28}$. Under hyperglycemic condition, the production of reactive oxygen species (ROS) is higher than that of the formation of antioxidants. This disruption of redox homeostasis elicits the peroxidation of proteins, lipids, and nucleic acids and further triggers the excessive activation of oxidative stress, which leads to insulin resistance, metabolic dysfunction, and even irreversible nerve cell death. Previous studies had demonstrated that chronic T2DM-induced excessive oxidative stress increased the vulnerability of peripheral nerves to diabetic neuropathy and cell death ${ }^{29,30}$. Furthermore, an upregulation of oxidative stress in diabetic rodents was found to increase the misfolding of myelinrelated proteins and alter myelin structure, which might have important implications for the demyelination process $^{31}$. Additionally, the disruption of SC metabolism under diabetic condition induced the rapid and robust accumulation of toxic oxygen free radicals and peroxides, which severely damaged axon and impaired vascular function ${ }^{32,33}$. Hence, decreasing the level of oxidative damage under diabetic condition is believed to be an important factor for reducing neuron demyelination.

Nrf2 acts as an important transcription factor for defending against oxidative damage and toxic insults ${ }^{34}$. A line of evidence suggests that the Nrf2-mediated cellular redox balance is regulated by Keap $1^{35-37}$. Importantly, Keap1/ Nrf2 signaling can drive cell proliferation and inhibit apoptosis through increasing various enzymatic antioxidants during diabetic neuropathy ${ }^{38}$. Thus, Nrf2/Keap1 signaling is an important regulatory pathway involved in defending against oxidative damage-induced neurodegenerative disease.

In the present study, we tried to explore whether aFGF has a significant effect on attenuating demyelination in T2DM and reveal its potential regulatory mechanism. Our findings indicated that aFGF ameliorates demyelination and upregulates myelin-related proteins and genes in chronic T2DM, and these effects were likely regulated by facilitating SC proliferation and migration and inhibiting its apoptosis. Unexpectedly, the anti-demyelinating and glial protective effects were correlated with aFGFmediated activation of Keap1/Nrf2 pathway, then further inhibiting excessive oxidative stress within SCs. These findings suggest that aFGF has therapeutic potential for preventing demyelination in patients with diabetes.

\section{Materials and methods \\ Reagents and antibodies}

aFGF was provided by the Key Laboratory of Biotechnology Pharmaceutical Engineering at Wenzhou Medical University, Wenzhou, Zhejiang, China. For the other reagents: hematoxylin and eosin (H\&E, Boster, AR1180), neutral resin (Solarbio, Cat\#G8590), phosphate buffered saline (PBS, Solarbio, P1010), Reactive Oxygen Species Assay Kit (ROS Assay Kit, Beyotime Biotechnology, S0033), bovine serum albumin (BSA, Beyotime Biotechnology, STO23), Dulbecco's modified Eagle Medium (DMEM, gibico, 11965092), Reverse transcription PCR Kit (RT-PCR Kit, TaKaRa, RR037A), 4'6-diamidino-2-phenylindoledihydrochloride (DAPI, Beycotime Biotechnology, C1006), Triton X-100 (Beycotime Biotechnology, T8200), TUNEL Apoptosis Detection Kit (Alexa Fluor 488, YE SEN Biotechnology, 40307ES60), Nuclear and Cytoplasmic Protein Extraction Kit (Beyotime Biotechnology, P0028). 
The related information of primary antibodies and their concentration used in this study was provided as follows: mouse polyclonal anti-FGF1 (Abcam, ab169748, 1:3000), anti-S100 (Santa Cruz, sc-53438, 1:500), mouse monoclonal anti-MBP (Abcam, ab62631, 1:3000), rabbit polyclonal antiMPZ (Abcam, ab31851, 1:3000), mouse monoclonal antiPCNA (Abcam, ab29, 1:500), rabbit monoclonal anti-Ki67 (Abcam,ab92742, 1:5000), rabbit monoclonal anti-Bax (CST, \#14796, 1:500), rabbit monoclonal anti-Bcl-2 (CST, \#4223, 1:500), rabbit polyclonal anti-Cleaved caspase-3 (Abcam, ab2302, 1:500), rabbit polyclonal anti-SOD2 (Abcam, ab13533, 1:5000), mouse monoclonal anti-HO-1 (Abcam, ab13248, 1:1000), rabbit monoclonal anti-NQO1 (Abcam, ab80588, 1:1000), rabbit monoclonal anti-Keap1 (CST, \#8047, 1:1000), rabbit monoclonal anti-Nrf2 (Abcam, ab76026, 1:5000), rabbit polyclonal anti-GAPDH (Millipore, AB2302, 1:10000), rabbit monoclonal anti-Histone H3 (Abcam, ab176842, 1:1000).

\section{Animal model and drug treatment}

Male C57BL/6J mice were bred in the Model Animal Research Center of Nanjing University (Nanjing, China). The process of inducing T2DM model was described previously $^{39}$. Briefly, C57BL/6 male mice, body weight of 20-30 g, were fed a high-fat diet (D12495; Research Diets) containing $10 \%$ fat, $1.5 \%$ cholesterol, $0.25 \%$ sodium cholate, $5 \%$ sucrose, and $83.25 \%$ basic feed for up to 12 weeks. Whereas, the mice in the control group were fed a standard diet (A500SL, Roles-Bio). Food intake and body weight were measured once a week. It should be emphasized that all the T2DM model animals used in the present study had met the following conditions: the blood glucose concentration $\geq 16.7 \mathrm{mmol} / \mathrm{L}$ and the body's weight over $20 \%$ of the mean value of the control. The protocols for animal care and use were conducted in accordance with the Guide for the Care and Use of Laboratory Animals from the National Institutes of Health. Before the start of the study, rats were allowed to acclimate to the facility for at least one week. Then, the total animals were randomly divided into control, T2DM and T2DM + aFGF groups, each group contained 10 mice. For the latter two groups, the high-fat diet mice were administered either with aFGF $(0.5 \mathrm{mg} / \mathrm{kg}$ body weight) or equal volume of physiologic saline via intraperitoneal (i.p.) injection every other day for one month. aFGF was dissolved in saline to achieve the working concentration of $20 \mu \mathrm{g} / \mathrm{mL}$. Later, the mice were euthanized, and sciatic nerve tissues were collected for the various pathology index analysis.

\section{Ultrastructural observation}

The ultrastructure of sciatic nerves in each group was observed under TEM. Briefly, samples were fixed in $2.5 \%$ glutaraldehyde for at least $48 \mathrm{~h}$. The tissues were then immersed in $1 \%$ osmium tetroxide solution and $1 \%$ uranyl acetate for $1 \mathrm{~h}$, respectively. Thereafter, the samples were dehydrated, embedded, and placed in baking box $\left(45^{\circ} \mathrm{C}\right)$ to keep dry for $72 \mathrm{~h}$. Lastly, the dry segments were cut and examined by TEM (H-600; HITACHI, Japan). Quantitative analyses were performed on five animals per group; each group randomly selected 100 myelinated axons from 10 images with the area of $30 \times 30 \mu \mathrm{m}^{2}$ to measure axon diameter and calculate g-ratio using Image J. The g-ratio was obtained by determining the inner axon diameter divided by the outer diameter of the myelin.

\section{Histological assessment}

After perfusion, the sciatic nerves in each group were harvested and fixed in a cold $4 \%$ paraformaldehyde at $4{ }^{\circ} \mathrm{C}$ for $24 \mathrm{~h}$. Afterward, the tissues were dehydrated in gradient concentrations of alcohol (70\%, 80\%, 90\%, 100\%) and embeded in paraffin. Next, the cross-sections of the nerve samples were cut in $8 \mu \mathrm{m}$ thicknesses for Hematoxylin-eosin (H\&E) staining. The images were acquired using a Nikon ECLIPSE 80i (Nikon, Japan).

\section{Real-time PCR}

Firstly, the collecting sciatic nerves were homogenized in TRIzol reagent. Then, total RNA was reversetranscribed into cDNA using Reverse Transcription Reagents Kit (TaKaRa, RR037A). The real-time PCR was conducted in a 7900HT Fast Real-Time PCR System using SYBR Green PCR Master Mix (Bio-Rad, Hercules, CA, USA). Lastly, the results were expressed as $2^{\wedge(-\Delta \Delta C t)}$. The primer sequence of Egr2, MBP, MPZ, Pmp22, and Sox10 genes for real-time PCR were listed in Table 1.

\section{Cell culture and treatment}

The RSC 96 cells (a rat SC line) were purchased from ScienCell Research Laboratories. At three passages, the cells were incubated in high-glucose (HG) environment to imitate pathogenic change in T2DM according to our previous report ${ }^{40}$. In brief, after seeding on a 6-plate well with a density of $5 \times 10^{5} / \mathrm{mL}$, SCs were placed into a humidified incubator overnight. Next day, the medium was changed to $30 \mathrm{mM}$ HG medium mixing with/without aFGF $(100 \mathrm{ng} / \mathrm{ml})$. Then, the cells were exposed in this concentration of $\mathrm{HG}$ condition for another $12 \mathrm{~h}$. We regarded HG plus aFGF as HG + aFGF group. The cells that only cultured in $\mathrm{HG}$ medium and normal medium were taken as HG group and control group, respectively.

For the preparation and transfections of small interfering RNA (siRNA), SC lines at 70-80\% confluence were infected with $200 \mathrm{pmol}$ Nrf2-siRNA or negative control siRNA (Genechem Co., Ltd., Shanghai) in serum-free medium. 24 h later, cells were cultured in HG + aFGF medium. Cells that were treated with scrambled siRNA control plasmid, together 
Table 1 Primers used for RT-PCR in this study.

\begin{tabular}{|c|c|c|c|}
\hline Gene & Prime sequence & Product size (bp) & Serial number \\
\hline \multirow[t]{2}{*}{$\beta$-actin } & F: GCAAGTGCTTCTAGGCGGACTG & 195 & NM_001101683.1 \\
\hline & R: CTGCTGTCACCTTCACCGTTCC & & \\
\hline \multirow[t]{2}{*}{ MBP } & F: AGTCCGACGAGCTACAGACCATC & 106 & XM_017338987.1 \\
\hline & R: TACTTGGAGCCGTGCCTCTGG & & \\
\hline \multirow[t]{2}{*}{ MPZ } & F: TCATCGAGATGGAGCTACGGAAGG & 89 & XM_008264187.2 \\
\hline & R: GGCGTTCTTGAGGCTGGTTCTG & & \\
\hline \multirow[t]{2}{*}{$\operatorname{Erg} 2$} & F: GTGGGGAGCGAGAACAATTA & 87 & XM_017338174.1 \\
\hline & R: GTTGTCGGTGAATTGGACCT & & \\
\hline \multirow[t]{2}{*}{ Pmp 22} & F: AGAGACAGCCATAAGGAGAACG & 96 & XM_017343068.1 \\
\hline & R: TGCAGACCACACCCTTCAT & & \\
\hline \multirow[t]{2}{*}{ Sox 10} & F: TCCAAAAACTAATCACAACAATCG & 141 & XM_008266894.2 \\
\hline & R: GAAGTGCAATTGGGATGAAAA & & \\
\hline
\end{tabular}

with aFGF and HG medium, were regarded as vehicle group. Specific silencing was identified by Western blotting (the best inhibition of Nrf2 expression was siRNA\#1 which was shown in Supplementary Fig. 1). The sequence of Nrf2-siRNA was shown below:

SiRNA\#1: 5'-CAG AAG AAC UGA UAG AGA U-3'

5'-AUC UCU AUC AGU UCU UCU G-3'

siRNA\#2: 5'-GAC AAG GAU GCU CGA GAC U-3'

5'-AGU CUC GAG CAU CCU UGU C-3'

SiRNA\#3: 5'-GUG ACU UGA AGA CUC UGA U-3'

5'-AUC AGA GUC UUC AAG UCA C-3'.

\section{Cell migration}

The RSC 96 SC lines were seeded in a six-plate well until they were grown to confluency. After serum-starved for $12 \mathrm{~h}$, the cells were scratched manually using a $1 \mathrm{~mL}$ plastic pipette tip. Then, each well was washed with PBS to remove unfastened cells and replaced with HG containing with/without aFGF according to the procedure mentioned above. Images of the wound closure or cell migration were taken immediately at time 0 and $12 \mathrm{~h}$ after the scratch using PowerShot G10 camera (Canon, Tokyo, Japan). The distance of scratches at $0 \mathrm{~h}$ and $12 \mathrm{~h}$ was quantified by Image $J$ software and marked as $A_{0}$ and $A_{12}$. The difference between $A_{12}$ and $A_{0}$ in each group was calculated to achieve the migration distance.

\section{Intracellular ROS detection}

The intracellular ROS production was detected using ROS Assay Kit (S0033, Beyotime, China). Briefly, after aspirating off the culture media, cells were incubated with $10 \mu \mathrm{M} 2^{\prime}, 7^{\prime}$-dichlorodihydrofluorescein diacetate (DCFHDA) in serum-free medium for $30 \mathrm{~min}$. Subsequently, the fluorescence images of cells were captured under the
Nikon ECLIPSE 80i (Nikon, Japan). Fluorescent intensity was quantified using Image J software.

\section{Western blotting}

Before conducting immunoblotting, we quantified protein concentration in each sample. Except for Nrf2 and Ki67, other biomolecules were extracted for detecting the total protein content. Briefly, homogenized tissue or living cells were lysed with ice-cold RIPA lysis buffer (Beyotime Biotechnology, P0013) containing $1 \mathrm{mmol} / \mathrm{L}$ PMSF, and $1 \mathrm{~g} / \mathrm{mL}$ protease inhibitors. Then, the samples were centrifugated at $16,099 \mathrm{~g}$ for $15 \mathrm{~min}$. The supernatant after centrifuging was used for the immunoblotting. For Nrf2 and Ki67, the whole proteins in cell or tissue were prepared using Nuclear and Cytoplasmic Protein Extraction Kit (Beyotime, Nantong, China). Afterward, the extracted proteins were processed for immunoblotting, including quantification of the protein concentrations, protein separation, transferring onto PVDF membranes, incubating with primary and second antibodies, and signals detection, which were all described previously ${ }^{41}$. The signal intensity of each protein was quantified using Image J software. The relative densities of the bands were normalized to GAPDH or Histone $\mathrm{H} 3$ and results were repeated at least three times.

\section{Immunofluorescence staining}

With regard to this experiment, the procedures were fully detailed in the previous description ${ }^{42}$. The tissue sections or cell samples were incubated with the primary antibodies and fluorescent-labeled secondary antibodies in $37^{\circ} \mathrm{C}$ condition for $2 \mathrm{~h}$ and $30 \mathrm{~min}$, respectively. The image acquisition was visualized with a confocal laser scanning microscope (Leica SP2, Mannheim, Germany). 


\section{TUNEL assay}

Cell apoptosis was detected by in situ terminal deoxynucleotidyl transferase-mediated dUTP-biotin nick end-labeling reaction (TUNEL) staining (Roche, 11684817910, Basel, Switzerland). According to the standard protocol, cells were fixed with $4 \%$ paraformaldehyde at $4{ }^{\circ} \mathrm{C}$ for $20 \mathrm{~min}$. Then, they were incubated with $1 \mu \mathrm{g} / \mathrm{ml}$ proteinase $\mathrm{K}$ solution for $15 \mathrm{~min}$. After washing with PBS three times for $15 \mathrm{~min}$, each well was incubated with $100 \mu \mathrm{L}$ TUNEL reaction mixture for $1 \mathrm{~h}$ at $37^{\circ} \mathrm{C}$. Subsequently, cells were stained with 4', 6-diamidino-2-pheny-lindole (DAPI, Beyotime, Shanghai, China) for $5 \mathrm{~min}$ and the images were acquired using a Nikon confocal laser microscope (Nikon, A1 PLUS, Tokyo, Japan).

\section{Statistical analysis}

All data were expressed as means \pm standard error of mean (SEM). Statistical comparisons of two or more treatment groups relative to the untreated control were examined by one-way analysis of variance (ANOVA) using the GraphPad Prism software Version 7 (GraphPad Software, Inc, San Diego, CA). For all analyses, a twosided $P$ value of less than 0.05 was deemed statistically significant.

\section{Results}

Chronic diabetes markedly inhibits aFGF expression in SCs

A reduction of endogenous aFGF was previously implicated in disease progression of T2DM patients ${ }^{43}$. Thus, we tried to detect aFGF level in the sciatic nerve tissues of T2DM mice. Western blotting showed that T2DM mice expressed lower levels of aFGF than control mice $(P<0.001$; Fig. 1a and b). Furthermore, we also found that the fluorescence intensity for aFGF was nearly colocalized with that for S100 (indicating mature SCs), suggesting that aFGF is mainly expressed in SCs (Fig. 1c and d). Next, we cultured SC lines under high-glucose (HG) conditions ( $30 \mathrm{mM}$ glucose) in vitro to mimic hyperglycemic conditions for diabetic patients, and measured the changes of aFGF expression at the indicated time points. As the result shown (Fig. $1 \mathrm{e}$ and $\mathrm{f}$ ), the expression of aFGF gradually increased as early as $3 \mathrm{~h}$ and peaked at $12 \mathrm{~h}$, after which aFGF level began to decrease. This phenomenon might indicate that SCs themselves secreted a certain amount of aFGF at an early time stage to resist the deteriorating external environment. Whereas, if this deterioration was continued for a prolonged time, SCs would suffer damage or apoptosis, leading to a decrease of aFGF production. In short, these experiments demonstrate that aFGF plays an important role in SCs under hyperglycemic conditions.

\section{aFGF attenuates diabetes-induced demyelination}

To determine the therapeutic effect of aFGF on remyelination, we firstly examined the morphological changes in the sciatic nerves of mice in each group using H\&E staining and TEM. Histopathological analysis of transverse sections of the nerve stumps in the control group had uniform, ordered, and dense myelination with structural integrity. In T2DM mice, massive nerve fibers exhibited vacuolar-like defects (Fig. 2a, marked with black arrows, indicating abnormal nerve fibers), and the percentage of these abnormal nerve fibers was noticeably more than that in the control group $(P<0.01$; Fig. $2 b)$. Moreover, morphological observation of the inner myelin in the T2DM group revealed many vacuoles, accompanied by onion bulb-shaped structures (shown in an enlarged image in Fig. 2c, defined as demyelination). In comparison, the axonal demyelination was largely alleviated in the T2DM + aFGF group, which was also confirmed by quantifying the proportion of demyelinated axons and analyzing the G-ratio (T2DM + aFGF vs. T2DM: $P<0.01$ for abnormal myelin and $P<0.05$ for G-ratio; Fig. $2 \mathrm{~d}$ and e). Next, we tested the expression of the myelin-associated proteins, MPZ and MBP, in each group through Western blotting. As illustrated in Fig. 2f-h, T2DM significantly reduced the levels of these proteins, but aFGF treatment appeared to attenuate the reduction of MPZ and MBP that observed in the nerves of T2DM + aFGF group. Similarly, the administration of exogenous aFGF largely activated myelination-related genes, including Egr 2, MBP, MPZ, Pmp 22 and Sox10, compared to the T2DM mice without any treatment (all $P<0.01$; Fig. 2i). Collectively, these data suggest that aFGF enhances remyelination in T2DM in vivo.

\section{aFGF facilitates SC proliferation and migration}

Substantial evidence has supported the notion that the enhanced proliferation and migration of SCs are a prerequisite for myelin regeneration ${ }^{44}$. To determine this effect in high-fat diet-fed T2DM model, we administered $0.5 \mathrm{mg} / \mathrm{kg}$ aFGF to T2DM mice by intraperitoneal injection every other day for 1 month. The degree of SC proliferation in each group was evaluated by immunostaining and immunoblotting. Double-immunostaining for Ki67 (to determine cell proliferation) and S100 (a marker of SCs) showed that Ki67 immunoreactivity was colocalized with the nuclei and distributed within the cytoplasm of S 100-labeled immature SCs in all three groups. Moreover, there were substantially fewer $\mathrm{Ki} 7^{+}$cells in T2DM mice than that in the normal mice, but aFGF treatment significantly reversed this trend $(P<0.05$; Fig. $3 \mathrm{a}$ and $\mathrm{b})$. Similarly, immunoblotting also exhibited that the protein levels of Ki67 and PCNA (another biomarker for cell proliferation) in sciatic nerves from T2DM mice 

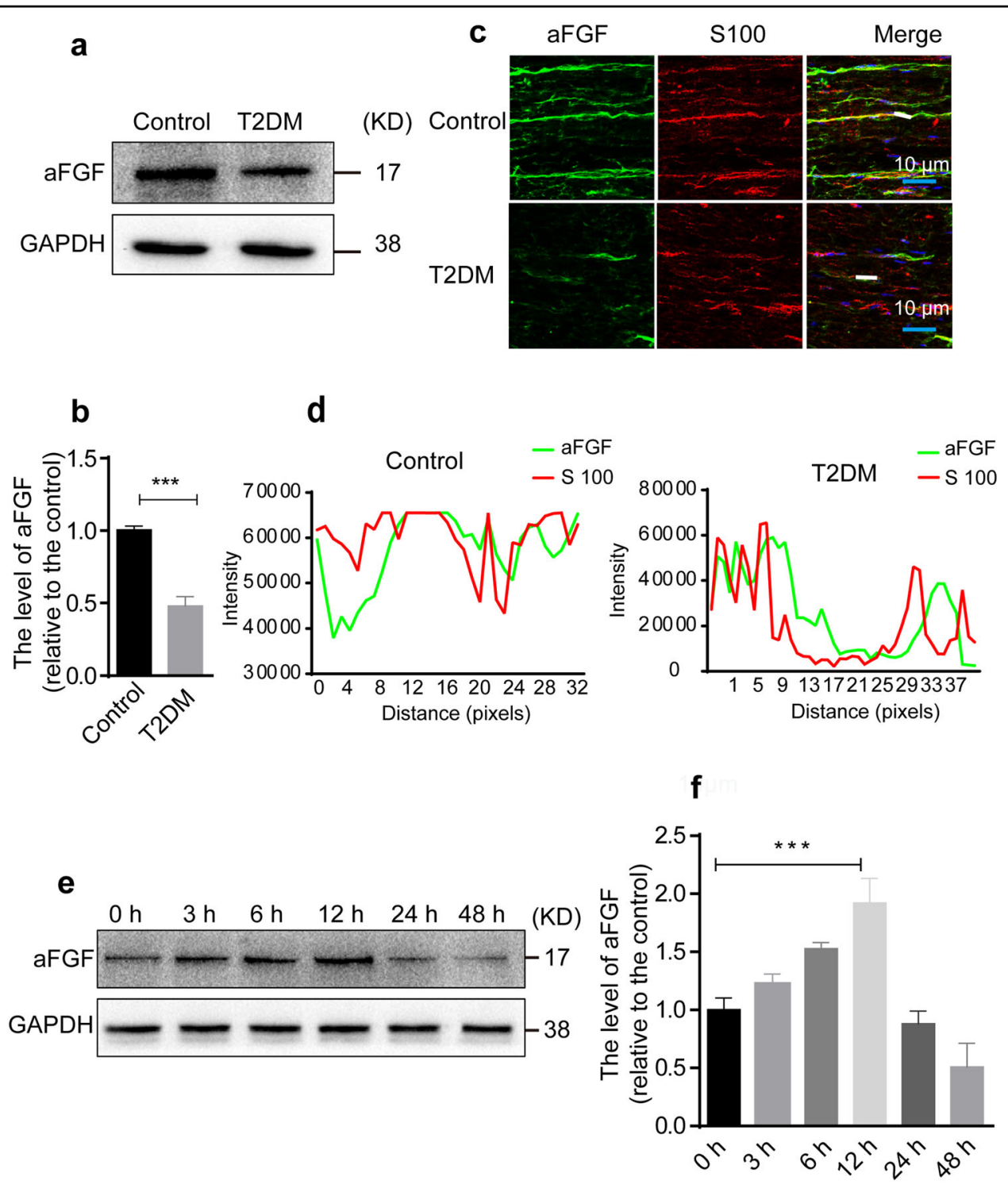

Fig. 1 Endogenous levels of aFGF in T2DM mice and SCs. $\mathbf{a}$, $\mathbf{b}$ Western blotting detected the content of aFGF in normal and T2DM mice. The result was repeated at least 3 times. c, $\mathbf{d}$ The longitudinal sections of sciatic nerves from normal and T2DM mice were double-immunostained with aFGF (green) and S100 (red) antibodies. The results of co-localization were analyzed by Image J software and GraphPad Prism. Scale bars represent $10 \mu \mathrm{m} . n=5$ mice per group with three images for each mouse. e, $\mathbf{f}$ Immunoblot and quantification data to show the aFGF level in SCs after cultured in $\mathrm{HG}(30 \mathrm{mM})$ medium for $3 \mathrm{~h}, 6 \mathrm{~h}, 12 \mathrm{~h}, 24 \mathrm{~h}$, or $48 \mathrm{~h}$. All data are the mean \pm SEM of three experiments. ${ }^{* * *} \mathrm{P}<0.001 \mathrm{vs}$. the T2DM group or the SCs exposing in $\mathrm{HG}$ for $12 \mathrm{~h}$.

were significantly lower than both of the T2DM + aFGF and control groups (all $P<0.05$; Fig. 3c-e).

Next, we used HG-treated SCs as an in vitro experimental model to evaluate whether aFGF could potentially facilitate their proliferation and migration. After $12 \mathrm{~h}$ of treatment, the SC proliferation rate was markedly reduced under HG condition but significantly rescued by aFGF treatment, which was reflected by the value of $\mathrm{Ki} 67^{+}$ positive cells and quantification of the band densities in each group by immunoblotting (Fig. $3 \mathrm{f}-\mathrm{j}$ ). Through a wound-healing assay, we found that the migratory ability of SCs was markedly impaired under HG conditions for culturing $12 \mathrm{~h}$. However, this inhibition was entirely reversed by aFGF treatment (Fig. 3k and 1). All of the above data suggest that aFGF plays a strong capacity to promote SC proliferation and migration.

\section{aFGF attenuates SC apoptosis}

To assess whether aFGF treatment promotes $\mathrm{SC}$ viability under hyperglycemic condition, we detected the apoptosis-related proteins via immunoblotting. As illustrated in Fig. 4a-c, the protein levels of cleaved caspase-3 


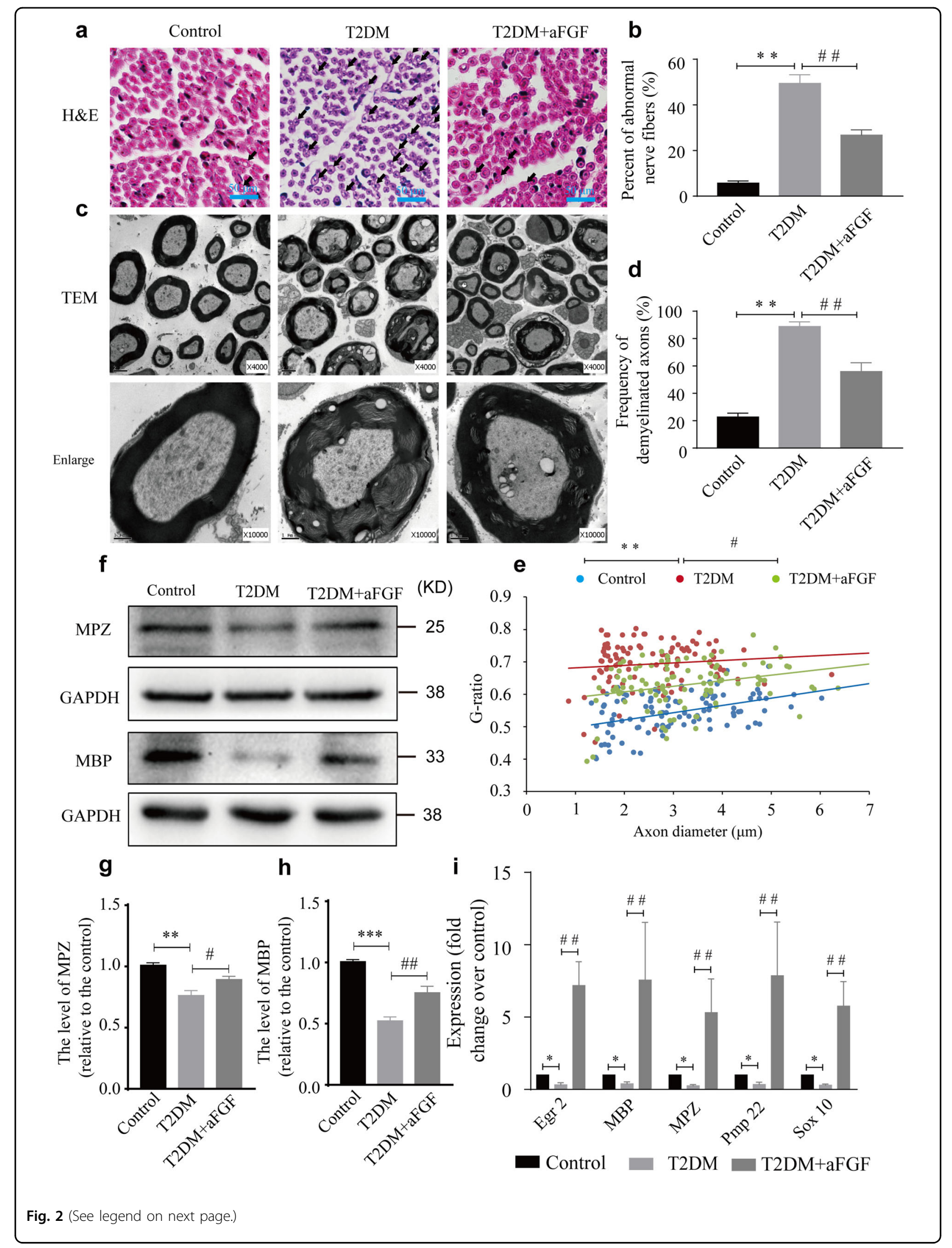


(see figure on previous page)

Fig. 2 aFGF promotes remyelination in T2DM model. $\mathbf{a}$, $\mathbf{b}$ Histomorphology images of H\&E staining from the sciatic nerves in the control, T2DM and T2DM + aFGF groups. The vacuolar-like structure of nerve fibers were indicated by green arrows. Their percent in each group were quantified with Image J software. Scale bar $=50 \mu \mathrm{m}$. c Representive TEM images in each group. Magnification of $\times 4000$ (scale bar $=2 \mu \mathrm{m}$, middle),

magnification of $\times 10,000$ (scale bar $=1 \mu \mathrm{m}$, bottom). $\mathbf{d}$, e A bar graph and scatter plot showing the frequency of abnormal myelin and the $\mathrm{g}$-ratio in each group from (c). $\mathbf{f}-\mathbf{h}$ Protein expressions of MPZ and MBP for the three experimental groups. $\mathbf{i}$ The expression of Egr2, MBP, MPZ, Pmp22, and SOX10 from different groups was detected via RT-PCR. Each gene expression value was normalized to $\beta$-actin. Data were the mean $\pm S E M, n=5$ animals per group for H\&E staining and TEM. For western blotting and RT-PCR, each experiment was independently repeated at least 3 times. ${ }^{*} P<$ $0.05,{ }^{* *} P<0.01,{ }^{* * * *} P<0.001$ vs. the control group; ${ }^{\#} P<0.05$, ${ }^{\# \#} P<0.01$ vs. the T2DM group.

in the sciatic nerve was significantly induced by T2DM. Whereas, the ratio of $\mathrm{Bcl}-2 / \mathrm{Bax}$ in T2DM exhibited reverse. In comparison, aFGF treatment largely reversed these changes (the statistical significance of differences of both the Bcl-2/Bax ratio and cleaved caspase-3 level between the T2DM + aFGF vs. T2DM groups was $P<$ 0.05.). Double-immunostaining result revealed that the cleaved caspase- 3 signal was nearly contained within the S100-positive SCs in all experimental groups (Fig. 4d). Moreover, the positive signal for cleaved caspase- 3 in each group was consistent with the results indicated by immunoblotting (Fig. 4e).

An in vitro apoptosis assay showed that there were significantly more TUNEL-positive nuclei in the HG group comparable to that in the control group $(P<0.001$; Fig. $4 \mathrm{f}$ and $\mathrm{g}$ ), but this increase was largely reduced after aFGF treatment (HG + aFGF vs. HG: $P<0.001$ ). Similarly, changes in expression of these apoptotic-related proteins in SCs were consistent with those in vivo (Fig. $4 \mathrm{~h}-\mathrm{j}$ ). These results further demonstrate that aFGF attenuates the apoptosis of SCs exposed to HG condition.

\section{aFGF treatment reduces hyperglycemia-induced oxidative stress in SCs}

Prolonged hyperglycemia is responsible for inducing oxidative stress as evident by increased ROS production and decreased antioxidant proteins, including SOD2, HO1 and $\mathrm{NQO} 1^{45,46}$. Here, we determined whether the aFGF administration could reverse hyperglycemia-induced oxidative stress activation. Compared with the control mice, the protein expressions of SOD2, HO-1, and NQO1 were significantly reduced in the sciatic nerve tissues of T2DM mice. However, aFGF treatment greatly reversed these changes (Fig. 5a-d).

Next, we compared the change of these proteins in control, HG and HG + aFGF groups. Consistent with the results in the animal model, the decreasing expression of SOD2, HO-1 and NQO1 in HG-cultured SCs were dramatically reversed after supplementation of the culture medium containing aFGF (Fig. 5e-h). In addition, ROS production was evaluated through DCFH-DA detection, a typical method used to measure ROS. As shown in Fig. 5i, $\mathrm{j}$, a lower level of ROS production was detected in
HG + aFGF group, when compared with the HG group. Collectively, these data indicate that aFGF has able to prevent hyperglycemia-induced oxidative stress activation both in vivo and in vitro.

\section{aFGF prevents hyperglycemia-induced oxidative stress through activating keap1/Nrf2 signaling}

Growing evidence has been shown that, under stress condition, such as chronic hyperglycemia, Keap1/ Nrf2 signaling played a pivotal role in regulating the antioxidant response ${ }^{47,48}$. Here, we found that the expression of Nrf2 in the cytoplasm and nucleus and Keap1 were significantly downregulated in T2DM mice but were greatly elevated after aFGF treatment (Fig. $6 a-d)$. Similar to the results in vivo, western blotting also revealed that the addition of aFGF significantly enhanced the intensities of the bands for Nrf2 and Keap1, compared with those in the HG group (Fig. 6e-h). Overall, our current results suggest that aFGF treatment significantly increases Keap1/Nrf2 signaling activation.

Silencing Nrf2 gene significantly abolishes aFGF biological effect on protecting SCs from HG-induced stress and apoptosis

To further determine the important role of aFGFmedicated Keap1/Nrf2 axis in protecting SC behavior, we silenced Nrf2 gene expression with the conditions of control, HG, HG + aFGF via transfecting Nrf2 siRNA into SCs. After $24 \mathrm{~h}$ transfection, there occurred a significant decrease of $\mathrm{Ki}^{+} 7^{+}$positive cells and migration distance (Fig. 7), but increase of Bcl-2/Bax ratio, Cleaved caspase-3 expression and TUNEL-positive cells (Fig. 8). In contrast, silencing the Nrf2 gene had no significant altered SC behavior with the conditions of control and HG (Figs. 7, 8).

In parallel, we also downregulated Nrf2 expression by using a novel and specific Nrf2 inhibitor, ML385 $(5 \mu \mathrm{M})^{49}$, prior to culture in HG medium containing aFGF. Similar to Nrf2 siRNA, ML385-treated cultures manifested a higher ROS generation (Fig. S2a, b) and cellular apoptosis (Fig. S2l-p) compared to only HG + aFGF treating SCs. Whereas, immunoblotting and the scratch test revealed that the expression of antioxidant and proliferationrelated proteins, as well as the speed of SC migration 


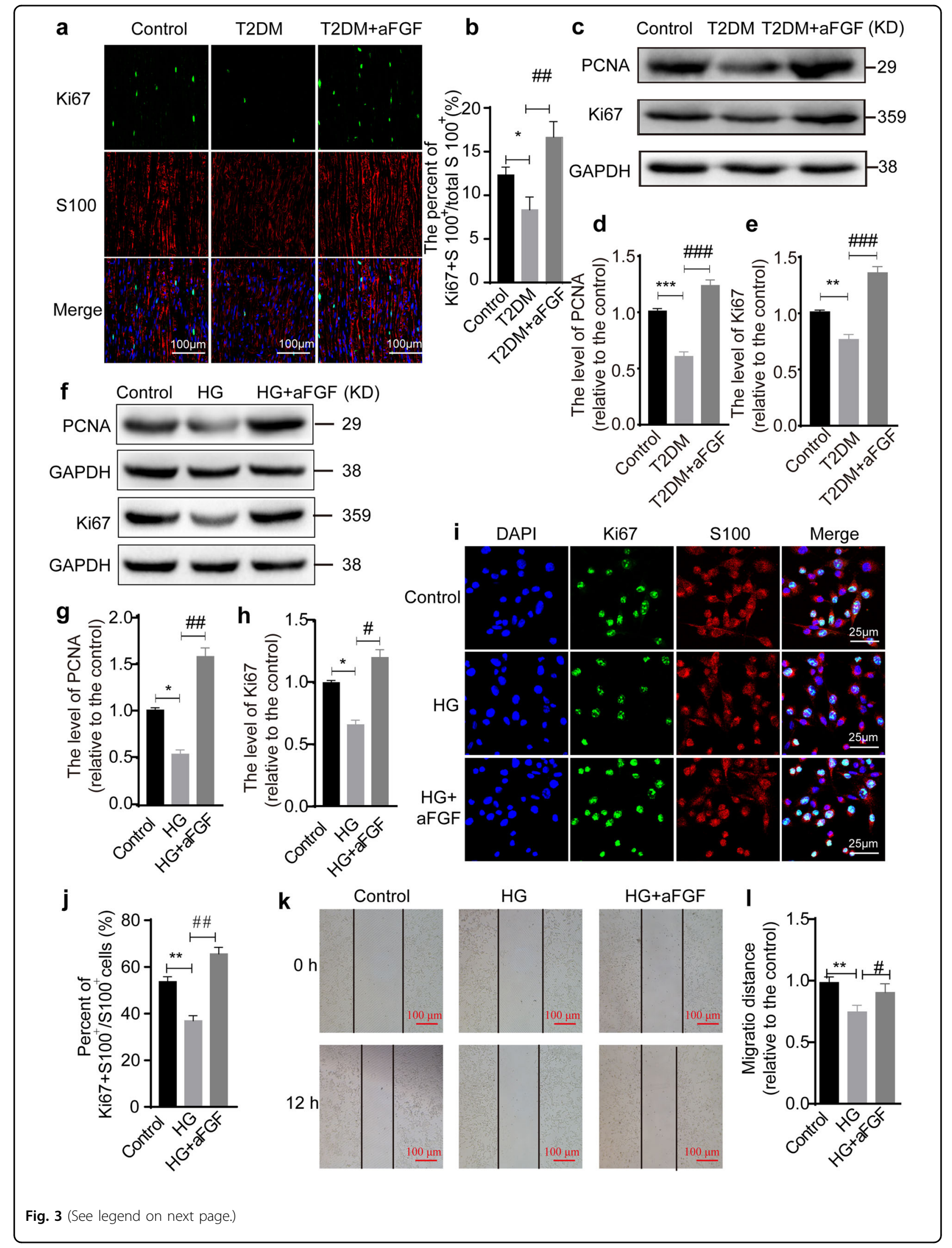




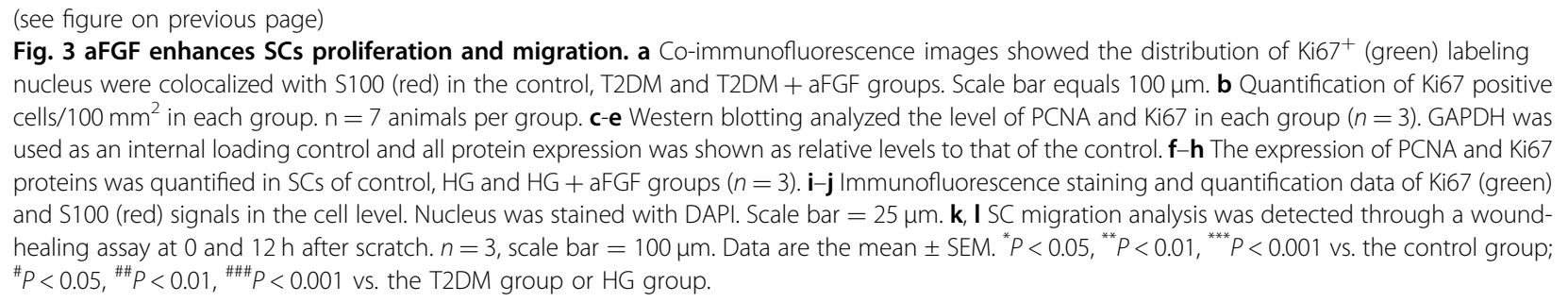

exhibited a reversed trend, i.e., the HG + aFGF+ML385 group had a lower level of antioxidant and proliferationrelated proteins (Fig. S2c-i) and slower speed of SC migration (Fig. S2j, k) than the HG + aFGF group. Hence, aFGF-medicated oxidative stress suppression and cellular behavior amelioration under HG condition are probably controlled by activating the Keap1/Nrf2 pathway in vitro.

\section{Discussion}

The new findings of the current study were as follows: (1) aFGF has a potential therapeutic effect in ameliorating myelin pathology and increasing the expression of myelinrelated genes in a prolonged T2DM model, (2) this beneficial effect is probably due to the aFGF-mediated increase in SC proliferation and migration and simultaneously attenuation of SC apoptosis, and (3) we determined that the molecular mechanism underlying aFGF controlling myelination and SC deficits under hyperglycemic conditions might be related to the Nrf2/Keap1 signaling-mediated suppression of oxidative stress. These findings highlight aFGF, as an available therapeutic strategy, ameliorates the pathological change of demyelination during the pathogenesis of T2DM and reveal its underlying molecular mechanism.

It has been shown that long-term exposure to elevated level of glucose would give rise to chronic demyelination, a notable characteristic of neuropathy that seriously damaged myelin, axons, and neuron cell bodies. In the PNS, axonal demyelination is closely associated with SC abnormalities, because myelin formation depends on SC development and maturation to wrap around axon. Besides, proliferating SCs have capable of providing abundant neurotrophins and oxygen to supporting axonal regeneration. Under diabetic condition, SCs are vulnerable to glucotoxicity-induced damage or apoptosis, which deteriorates myelin splitting and myelin shedding, leading to axonal dysfunction and demyelination. Previous reports have shown that hyperglycemia-induced SC dysfunction and toxicity were associated with metabolic alterations and/or inflammatory aggravation ${ }^{50,51}$. Increasing evidence has focused on altering $\mathrm{SC}$ behavior to reverse progressive myelin degeneration. For instance, converting the $\mathrm{SC}$ phenotype from a denervated state to an elongated and bipolar morphology promotes the shortening of remyelination ${ }^{52}$. Overexpression of Neuregulin-1 type I (NRG1-I) was found to be required for SC differentiation, which further accelerates remyelination in chronic demyelinating neuropathy ${ }^{53}$. Moreover, SC proliferation and dedifferentiation are requisite for myelin generation and maintenance ${ }^{54}$. Therefore, we try to restore myelin disorganization and maintain normal myelin morphology through improving SC plasticity and activating intrinsic reprogramming in SCs.

aFGF plays multiple regulatory roles in the biological processes of cellular survival, proliferation, migration, and plasticity. Previous our and the others' works had confirmed that aFGF possessed striking neuroprotective and neuroregenerative effects in the nervous system $^{55,56}$. Increasing evidence has indicated that aFGF could prevent the occurrence and development of a number of diabetic complication, including diabetic cardiomyopathy, hepatic damage, and nephropathy ${ }^{57-59}$. More importantly, Suh. et al first reported that only a single injection of recombinant aFGF provoked a sustained decrease in glucose and insulin sensitization in diabetic hyperglyce$\mathrm{mia}^{23}$. This exciting discovery was also verified in another report that utilized a common aFGF at $1 / 10$ the dose to treat a rodent model of $\mathrm{T} 2 \mathrm{DM}^{22}$. Accordingly to these reports, we speculate that aFGF plays a positive role in improving $\mathrm{SC} /$ myelin pathology in diabetic hyperglycemia.

To verify this hypothesis, high-fat diet-fed T2DM mice and SCs exposed to high glucose were treated with/ without aFGF. At predetermined times, pathology parameters in the sciatic nerve or SCs were assessed. As expected, severe demyelination and SC apoptosis in the T2DM model was dramatically reversed after aFGF administration. Simultaneously, SC proliferation and migration increased as well. These data indicated that the injection of aFGF reduced demyelination and increased the expression level of myelin-associated proteins, which was critically related to aFGF-induced enhancement of survival, proliferation and migration in SCs. Nevertheless, the potential mechanism that regulates the effect of aFGF in ameliorating progressive demyelination in this condition remains unknown. 

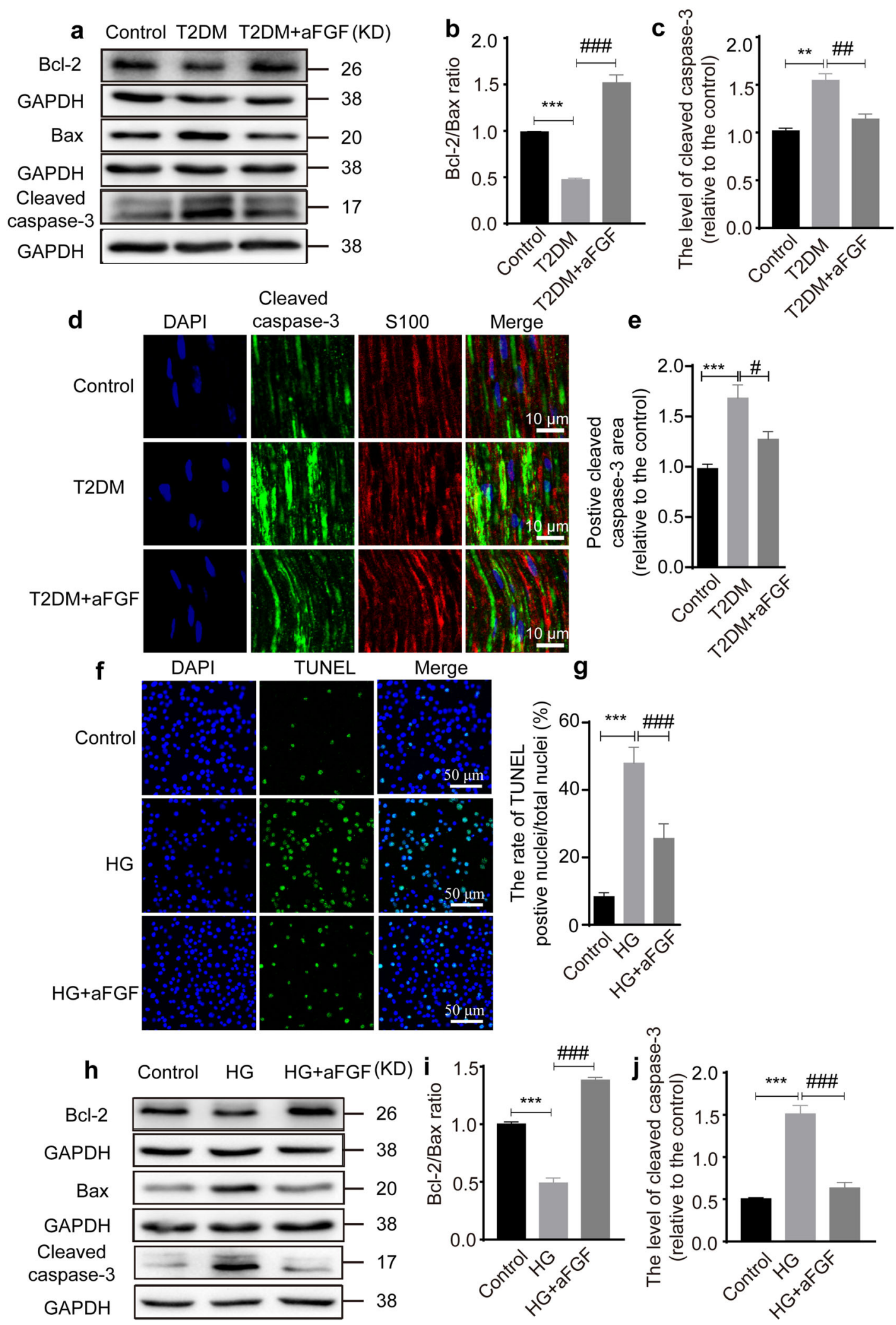

Fig. 4 (See legend on next page.) 
(see figure on previous page)

Fig. 4 aFGF inhibits SCs apoptosis. a-c Representative western blotting bands plus the corresponding statistical results of Bax, Bcl-2 and cleaved caspase-3 in control, T2DM and T2DM + aFGF groups. Data were repeated three times. $\mathbf{d}$ Co-staining of cleaved caspase-3 (green) and S100 (red) in each group. Nucleus were labeled with DAPI (blue). Scale bar equals $10 \mu \mathrm{m}$. e Quantification of positive cleaved caspase-3 area from (d). $n=7$ animals per group. $\mathbf{f}$ Representative SC apoptosis images at $12 \mathrm{~h}$ after HG. Scale bar $=50 \mu \mathrm{m}$. $\mathbf{g}$ Quantification of the percentage of TUNEL-positive $\mathrm{SCs}$ from (f). This test was independently repeated 5 times. $\mathbf{h}$ Protein level of Bax, BCl-2 and cleaved caspase-3 among the Control, HG, HG $+\mathrm{aFGF}$ groups. $\mathbf{i}$, j Densitometric analyses of Western blotting from $(\mathbf{h})$. This experiment was repeated in triplicate. Data are the mean \pm SEM, ${ }^{* *} P<0.01,{ }^{* * * *} P<$ 0.001 vs. the control group; ${ }^{\#} P<0.05,{ }^{\# \#} P<0.01,{ }^{\# \# \#} P<0.001$ vs. the T2DM group or HG group.

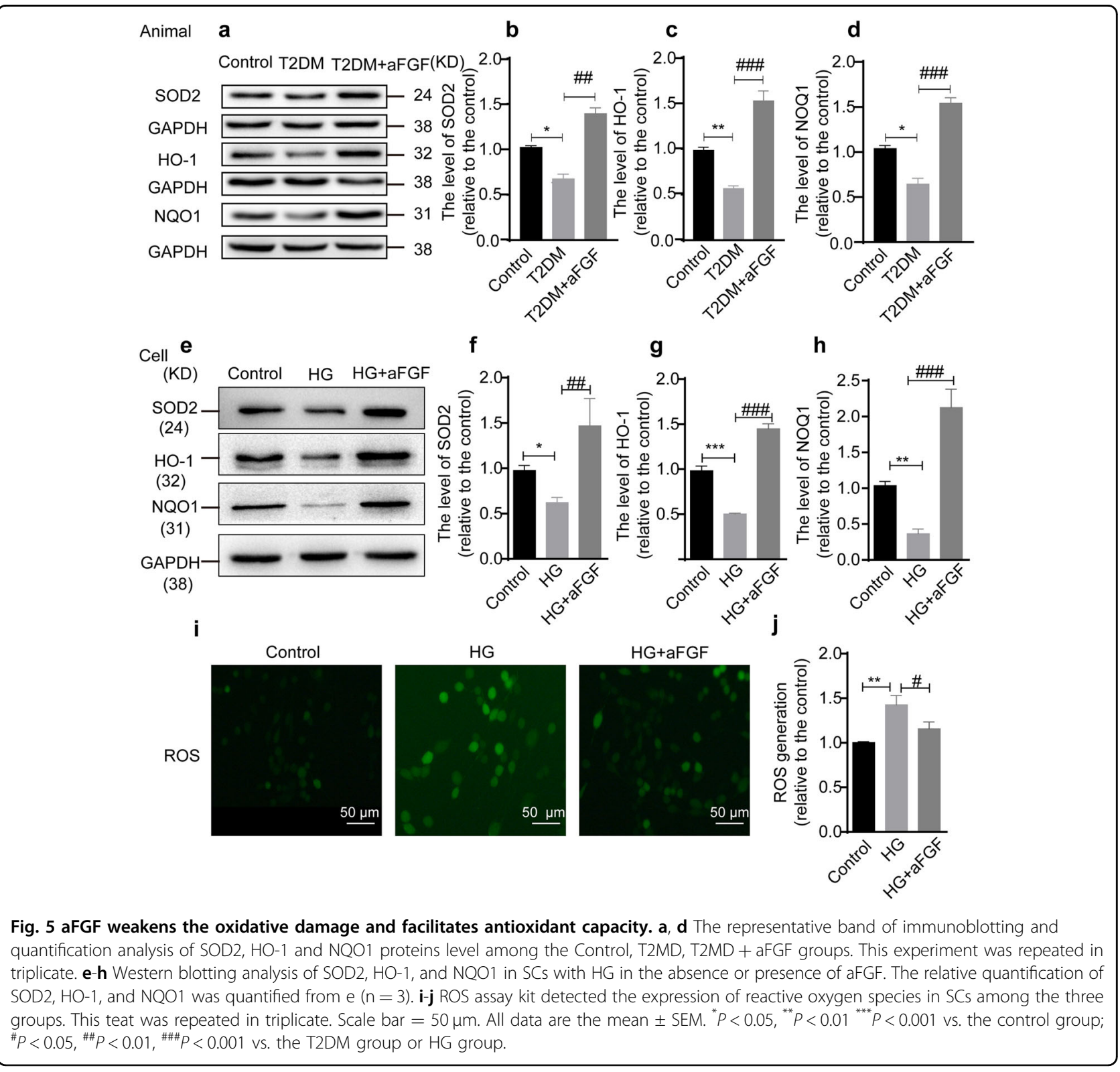

Recent evidence has demonstrated that increased oxidative stress and ROS are the leading causative factors driving the pathogenesis of $\mathrm{T}_{2} \mathrm{DM}^{60-62}$. During the development of T2DM, the cells and tissue of the body are exposed to hyperglycemic condition. Such elevated glucose levels promote progressive oxidative stress activation, resulting in excess ROS production. The overproduction of ROS can alter cell destiny and impair 


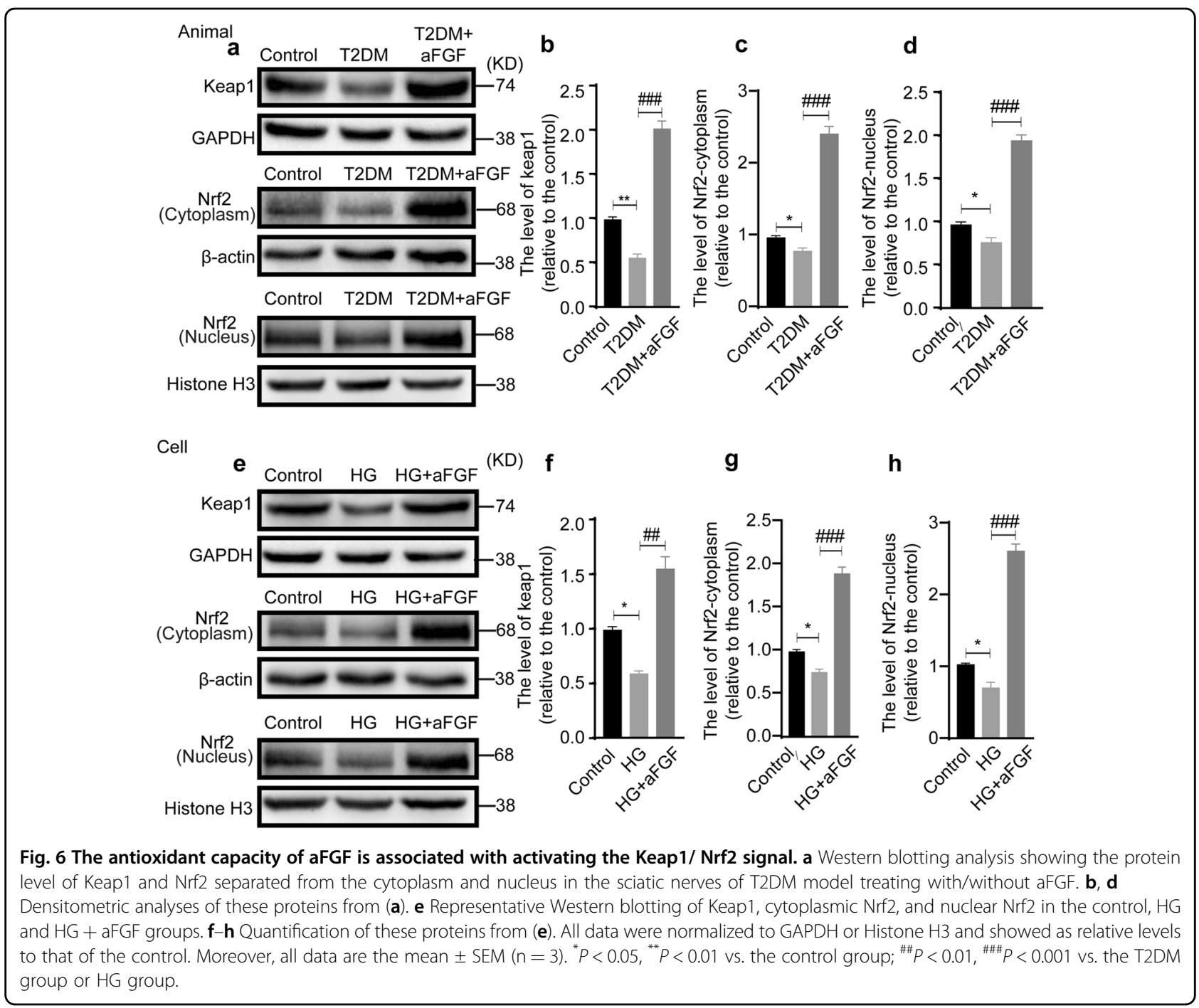

the structure and function of body tissues, such as the peripheral nerve ${ }^{63}$. Hamilton. et al reported that oxidative stress was closely associated with the reduced myelination in peripheral neuropathies ${ }^{31}$. Moreover, accumulating evidence has shown that oxidative stress could reduce SC survival and induce SC apoptosis ${ }^{64,65}$. However, we still unknown whether the beneficial effect of aFGF in preventing myelin pathology and protecting SCs from HGinduced apoptosis was related to excessive oxidative stress activation. Presently, we documented that the sciatic nerves in T2DM mice expressed low levels of the antioxidative related proteins, including SOD2, HO-1, and NOQ1, but aFGF significantly reversed this trend. Furthermore, the results of an in vitro experiment showing that aFGF regulated SC behavior under high-glucose conditions was consistent with the in vivo results. These data indicate that the aFGF-induced reversal of SC dysfunction and demyelination may be associated with its suppression of excessive oxidative stress activation.
The Keap1/Nrf2 pathway is regarded as the primary mediator of oxidative stress responses in a wide array of disease models ${ }^{66}$. It is well known that Nrf2 can be dissociated from the Keap1/Nrf2 complex upon oxidative stress and translocates into the cell nucleus to induce the transcription of a serious of antioxidant defense genes that contribute to the resistance against excitotoxic and oxidative insults in neurological disorders ${ }^{67-69}$. Activation of the Nrf2 signaling cascade reaction via antioxidant substances is also found to attenuate $\mathrm{H}_{2} \mathrm{O}_{2}$-induced mitochondrial dysfunction and SC apoptosis ${ }^{70}$. Moreover, we previously revealed that a reduction in peripheral nerve injury (PNI)-induced oxidative damage could be achieved through fibroblast growth factor 21 (FGF21)mediated Nrf2 signaling activation ${ }^{64}$. These findings indicate that Keap1/Nrf2 signaling may be the main upstream regulator that controls oxidative stress-induced demyelination in T2DM. To confirm this hypothesis, the protein expression of $\mathrm{Nrf} 2$ and Keap 1 in each group was 


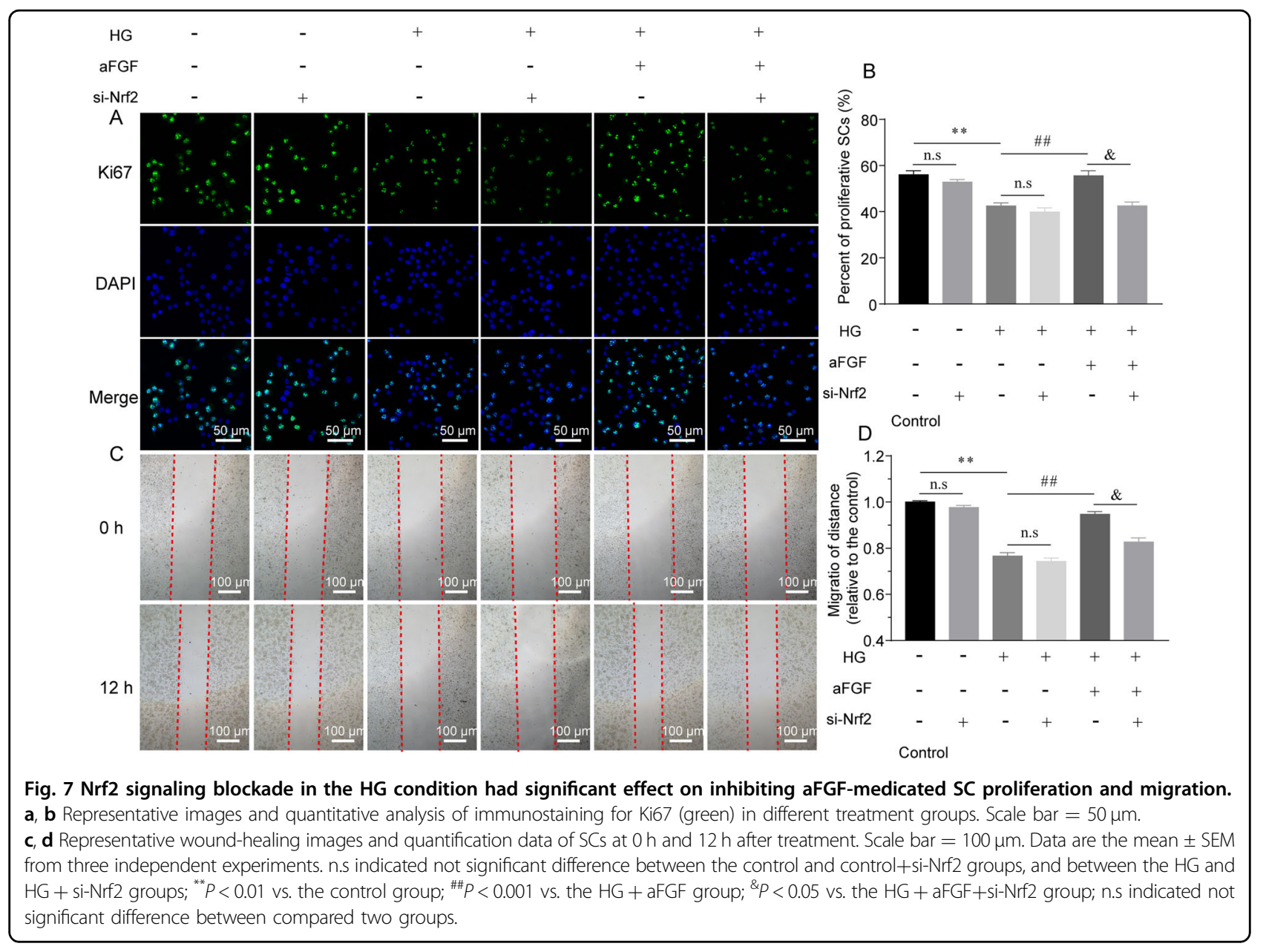

detected using immunoblotting. The results showed that the decrease in Nrf2 and Keap1 in the T2DM model was reversed after direct treatment with aFGF. These findings were consistent with our results collected from a SC line cultured under high-glucose condition. Additionally, the suppression of Nrf2 activation through genetic or pharmacological methods in vitro significantly restored oxidative stress, resulting in the decrease of SC survival. These results suggest that the Keap1/Nrf2 axis maybe act as the underlying mechanism for guiding aFGF to attenuate excessive oxidative damage in SCs.

Nevertheless, aFGF-mediated SC protection and neuroplasticity should also be regulated by other signaling cascades. For instance, endogenous aFGF has been shown to specifically express in motor and sensory neurons, which was participated in neurite outgrowth and axonal regeneration via activating MEK-ERK1/2-STAT3-Egr1 pathway after sciatic nerve damage ${ }^{21,71}$. In vitro, the usage of aFGF was required to facilitate proliferation of primary SCs predominantly through JAK/STAT3 transduction pathway $^{72}$. Likewise, aFGF-dependent Rho-GTPase suppression also plays an essential role in decreasing the growth inhibitory molecules, and therefore enhancing intrinsic ability of regeneration in the injured spinal $\operatorname{cord}^{73}$. Thus, we speculate all of these underlying mechanisms mentioned above are also participate in aFGF benefit effects on exerting remyelination in T2DM.

Another important issue is selecting the suitable time period of SCs culturing in HG in vitro that matches the similar characteristics of SCs in vivo hyperglycemia condition. In our studies, we observed that the aFGF expression was upregulation at early time and subsequently became downregulation at later time. This expressing change is consistent with the findings of diabetes-induced alterations in the expression of aFGF in vivo model $^{58,74}$. Thus, as for the prolonged T2DM mice, we detected the aFGF expression in their tissues was less than that of the normal mice. The reason is that SCs are susceptible to HG infiltration. If SCs exposing in the HG in vitro is not too long, they triggered a serious of pathological signaling events, including increased ROS levels, weak antioxidant capacity, and depolarization of mitochondria, which manifested the similar characteristics of SCs in vivo hyperglycemia condition ${ }^{75,76}$. 


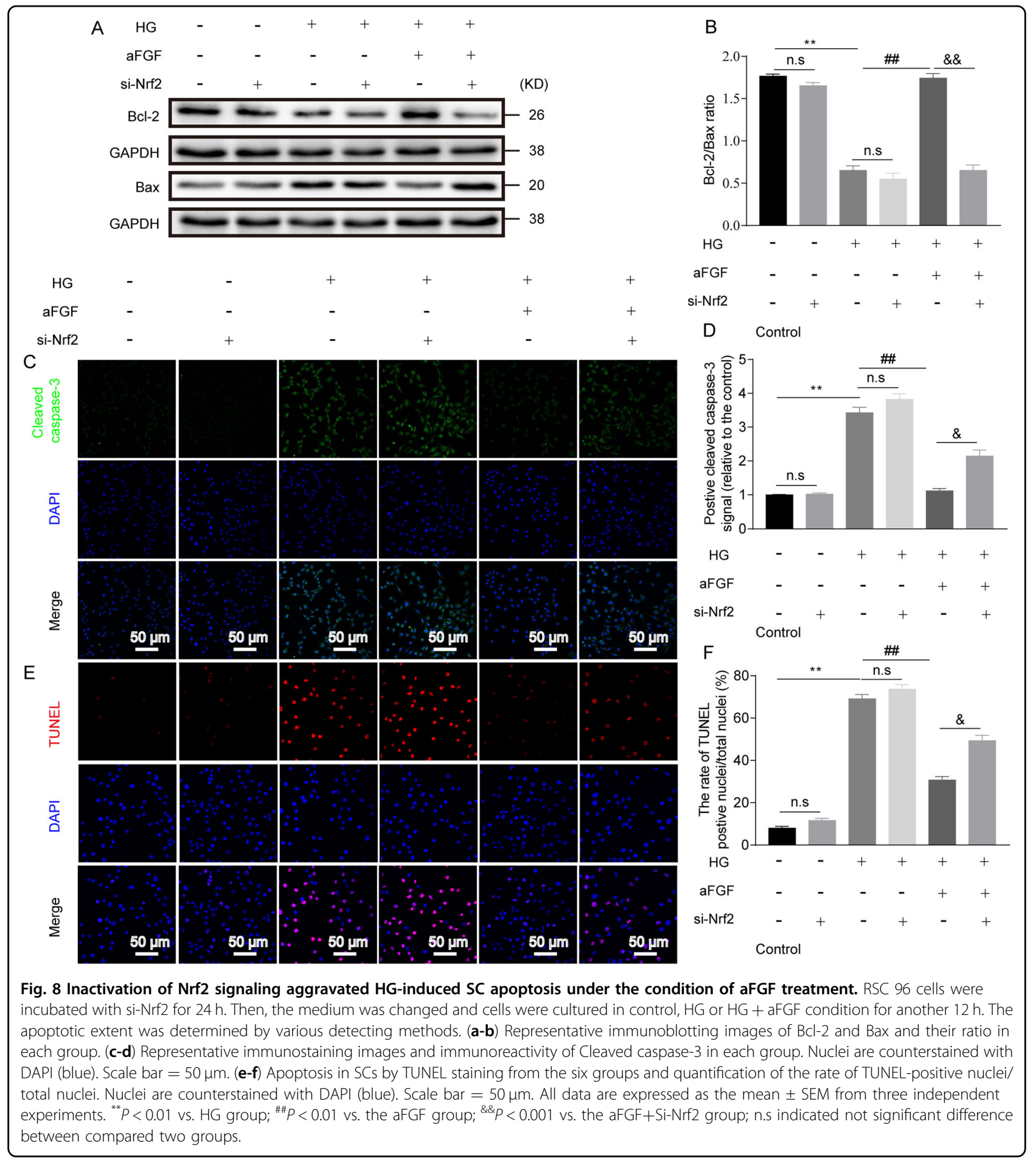

Thus, during this period, SCs cultured in HG are suitable for modeling hyperglycemia in T2DM. However, if culturing in HG is too long, SCs display acute metabolic disorder and dysfunction, leading to their death or disintegration ${ }^{77}$. This situation is not only inappropriate to model hyperglycemia in T2DM, but also totally unsuited for the experimental progression. But it is should be noted that the specific time point that distinguishes the physiological state of SCs culturing in HG in vitro from exposing in chronic hyperglycemia in vivo is still unknown. This issue needs to be explored further.

In summary, this study demonstrated that the longterm administration of aFGF facilitated a marked increase in SC proliferation and migration, and suppressed SC 
apoptosis to ameliorate disordered demyelination in T2DM mouse model. Furthermore, we also revealed that the molecular mechanisms of these beneficial effects were related to the aFGF-mediated excessive oxidative stress blockade, which was seemed to regulate via the activation of the Keap1/Nrf2 signaling (a schematic diagram is shown in Fig. S3). All of these findings indicate that aFGF is a promising therapeutic agent for preventing the progression of chronic demyelinating diseases via reducing diabetes-induced cellular stress.

\section{Author contributions}

X.J., W.J., and L.R. conceived and designed the research work. L.R., W.B., L.D., Y.L., and C.X. performed the experiments. L.R., W.Y., W.B., and L.D. conducted statistical analysis and wrote the paper. W.C., Y.L., L.P., Y.Y., Z.H., X.L., and L.X. provided assistance with experiments. All authors discussed the manuscript drafting

\section{Funding}

This study was partially supported by a research grant from the National Natural Science Funding of China $(81802238,81801233,81722028,81801245$, 81870842), Zhejiang Provincial Natural Science Foundation of China (WQ20H170001, R18H150001, Q18H090037, LY17H090017), Research Unit of Research and Clinical Translation of Cell Growth Factors and Diseases of Chinese Academy of Medical Science (2019RU010).

\section{Author details}

'Department of Hand Surgery and Peripheral Neurosurgery, The First Affiliated Hospital and School of Pharmaceutical Sciences, Wenzhou Medical University, 325000 Wenzhou, Zhejiang, China. ${ }^{2}$ Research Center, Affiliated Xiangshang Hospital, Wenzhou Medical University, 315700 Ningbo, Zhejiang, China.

${ }^{3}$ School of Chemistry, Sun Yat-sen University, 510275 Guangzhou, Guangdong, China

\section{Data availability}

The datasets used and/or analyzed during the current study are available from the corresponding author on reasonable request.

\section{Conflict of interest}

The authors declare that they have no conflict of interest.

\section{Publisher's note}

Springer Nature remains neutral with regard to jurisdictional claims in published maps and institutional affiliations.

Supplementary information The online version contains supplementary material available at https://doi.org/10.1038/s41419-021-03407-2.

Received: 21 August 2020 Revised: 16 December 2020 Accepted: 17 December 2020

Published online: 21 January 2021

\section{References}

1. Jaacks, L. M., Siegel, K. R., Gujral, U. P. \& Narayan, K. M. Type 2 diabetes: a 21st century epidemic. Best. Pract. Res. Clin. Endocrinol. Metab. 30, 331-343 (2016).

2. Hod, M. et al. The International Federation of Gynecology and Obstetrics (FIGO) Initiative on gestational diabetes mellitus: a pragmatic guide for diagnosis, management, and care. Int. J. Gynaecol. Obstet. 131, S173-211 (2015).

3. Collaboration, N. C. D. R. F. Worldwide trends in diabetes since 1980: a pooled analysis of 751 population-based studies with 4.4 million participants. Lancet 387, 1513-1530 (2016).
4. Nathan, D. M. Diabetes: advances in diagnosis and treatment. Jama $\mathbf{3 1 4}$, 1052-1062 (2015).

5. Jangam, S. R., Hayter, G. \& Dunn, T. C. Individuals with type 1 and type 2 diabetes mellitus trade increased hyperglycemia for decreased hypoglycemia when glycemic variability is not improved. Diabetes Ther.: Res. Treat. Educ. Diabetes Relat. Disord. 9, 395-402 (2018).

6. Javed, S., Petropoulos, I. N., Tavakoli, M. \& Malik, R. A. Clinical and diagnostic features of small fiber damage in diabetic polyneuropathy. Handb. Clin. Neurol. 126, 275-290 (2014).

7. Hayden, M. R., Grant, D. G., Aroor, A. R. \& DeMarco, V. G. Empagliflozin ameliorates type 2 diabetes-induced ultrastructural remodeling of the neurovascular unit and neuroglia in the female db/db mouse. Brain Sci. 9, https://doi. org/10.3390/brainsci9030057 (2019).

8. Zanazzi, G., Arshad, M., Maurer, M. S., Brannagan, T. H. 3rd \& Tanji, K. Demyelinating neuropathy in a patient treated with revusiran for transthyretin (Thr60Ala) amyloidosis. J. Clin. Neuromuscul. Dis. 20, 120-128 (2019).

9. Bansagi, B. et al. Multifocal demyelinating motor neuropathy and hamartoma syndrome associated with a de novo PTEN mutation. Neurology 90, e1842-e1848 (2018)

10. Jessen, K. R. \& Mirsky, R. The origin and development of glial cells in peripheral nerves. Nat. Rev. Neurosci. 6, 671-682 (2005).

11. Brierley, C. M. et al. Remyelination of demyelinated CNS axons by transplanted human Schwann cells: the deleterious effect of contaminating fibroblasts. Cell Transplant. 10, 305-315 (2001).

12. Dyck, P. J. \& Giannini, C. Pathologic alterations in the diabetic neuropathies of humans: a review. J. Neuropathol. Exp. Neurol. 55, 1181-1193 (1996).

13. Dey, I. et al. Diabetic Schwann cells suffer from nerve growth factor and neurotrophin-3 underproduction and poor associability with axons. Glia 61, 1990-1999 (2013)

14. Su, W. F. et al. Overexpression of P2X4 receptor in Schwann cells promotes motor and sensory functional recovery and remyelination via BDNF secretion after nerve injury. Glia 67, 78-90 (2019).

15. Dusart, I., Marty, S. \& Peschanski, M. Demyelination, and remyelination by Schwann cells and oligodendrocytes after kainate-induced neuronal depletion in the central nervous system. Neuroscience 51, 137-148 (1992).

16. Georgiou, V. \& Gkretsi, V. The role of fibroblast growth factors and their receptors in gliomas: the mutations involved. Rev. Neurosci. 30, 543-554 (2019).

17. Chiu, I. M., Touhalisky, K. \& Baran, C. Multiple controlling mechanisms of FGF1 gene expression through multiple tissue-specific promoters. Prog. Nucleic Acid Res. Mol. Biol. 70, 155-174 (2001)

18. Pirou, C. et al. FGF1 protects neuroblastoma SH-SY5Y cells from p53dependent apoptosis through an intracrine pathway regulated by FGF1 phosphorylation. Cell Death Dis. 8, e3023 (2017).

19. Tsai, M. J. et al. Acidic FGF promotes neurite outgrowth of cortical neurons and improves neuroprotective effect in a cerebral ischemic rat model. Neuroscience 305, 238-247 (2015).

20. Cuevas, P., Carceller, F. \& Gimenez-Gallego, G. Acidic fibroblast growth factor prevents post-axotomy neuronal death of the newborn rat facial nerve. Neurosci. Lett. 197, 183-186 (1995).

21. Laird, J. M., Mason, G. S., Thomas, K. A., Hargreaves, R. J. \& Hill, R. G. Acidic fibroblast growth factor stimulates motor and sensory axon regeneration after sciatic nerve crush in the rat. Neuroscience 65, 209-216 (1995).

22. Scarlett, J. M. et al. Central injection of fibroblast growth factor 1 induces sustained remission of diabetic hyperglycemia in rodents. Nat. Med. 22, 800-806 (2016).

23. Suh, J. M. et al. Endocrinization of FGF1 produces a neomorphic and potent insulin sensitizer. Nature 513, 436-439 (2014).

24. Jonker, J. W. et al. A PPARgamma-FGF1 axis is required for adaptive adipose remodelling and metabolic homeostasis. Nature 485, 391-394 (2012).

25. Sun, K. \& Scherer, P. E. The PPARgamma-FGF1 axis: an unexpected mediator of adipose tissue homeostasis. Cell Res. 22, 1416-1418 (2012).

26. van der Kooij, M. A. et al. Chronic social stress-induced hyperglycemia in mice couples individual stress susceptibility to impaired spatial memory. Proc. Natl Acad. Sci. USA 115, E10187-E10196 (2018).

27. Rehman, K. \& Akash, M. S. H. Mechanism of generation of oxidative stress and pathophysiology of type 2 diabetes mellitus: how are they interlinked? J. Cell. Biochem. 118, 3577-3585 (2017).

28. Ma, X. et al. The pathogenesis of diabetes mellitus by oxidative stress and inflammation: its inhibition by Berberine. Front. Pharmacol. 9, 782 (2018). 
29. Maiese, K., Chong, Z. Z. \& Shang, Y. C. Mechanistic insights into diabetes mellitus and oxidative stress. Curr. Medicinal Chem. 14, 1729-1738 (2007).

30. Newsholme, P., Cruzat, V. F., Keane, K. N., Carlessi, R. \& de Bittencourt, P. I. Jr. Molecular mechanisms of ROS production and oxidative stress in diabetes. Biochem. J. 473, 4527-4550 (2016).

31. Hamilton, R. T. et al. Elevated protein carbonylation, and misfolding in sciatic nerve from $\mathrm{db} / \mathrm{db}$ and Sod1(-/-) mice: plausible link between oxidative stress and demyelination. PlOS ONE 8, e65725 (2013).

32. Goncalves, N. P. et al. Schwann cell interactions with axons and microvessels in diabetic neuropathy. Nat. Rev. Neurol. 13, 135-147 (2017).

33. Eckersley, L., Ansselin, A. D. \& Tomlinson, D. R. Effects of experimental diabetes on axonal and Schwann cell changes in sciatic nerve isografts. Brain Res. Mol. Brain Res. 92, 128-137 (2001).

34. Harder, B. et al. Molecular mechanisms of Nrf2 regulation and how these influence chemical modulation for disease intervention. Biochem. Soc. Trans. 43, 680-686 (2015).

35. O'Connell, M. A. \& Hayes, J. D. The Keap1/Nrf2 pathway in health and disease: from the bench to the clinic. Biochem. Soc. Trans. 43, 687-689 (2015).

36. Hayes, J. D., Chowdhry, S., Dinkova-Kostova, A. T. \& Sutherland, C. Dual regulation of transcription factor Nrf2 by Keap1 and by the combined actions of beta-TrCP and GSK-3. Biochem. Soc. Trans. 43, 611-620 (2015).

37. Paunkov, A., Chartoumpekis, D. V., Ziros, P. G. \& Sykiotis, G. P. A bibliometric review of the Keap1/Nrf2 Pathway and its related antioxidant compounds. Antioxidants 8, https://doi.org/10.3390/antiox8090353 (2019).

38. Kumar, A. \& Mittal, R. Nrf2: a potential therapeutic target for diabetic neuropathy. Inflammopharmacology 25, 393-402 (2017).

39. Winzell, M. S. \& Ahren, B. The high-fat diet-fed mouse: a model for studying mechanisms and treatment of impaired glucose tolerance and type 2 diabetes. Diabetes 53, S215-219 (2004)

40. Li, R. et al. NGF attenuates high glucose-induced ER stress, preventing Schwann cell apoptosis by activating the PI3K/Akt/GSK3beta and ERK1/2 pathways. Neurochem. Res. 42, 3005-3018 (2017)

41. Li, R. et al. Heparin-poloxamer thermosensitive hydrogel loaded with bFGF and NGF enhances peripheral nerve regeneration in diabetic rats. Biomaterials 168, 24-37 (2018).

42. Li, R. et al. Single injection of a novel nerve growth factor coacervate improves structural and functional regeneration after sciatic nerve injury in adult rats. Exp. Neurol. 288, 1-10 (2017).

43. Crunkhorn, S. Metabolic disease: FGF1 stops diabetes in its tracks. Nat. Rev. Drug Discov. 15, 456 (2016).

44. Liu, B. et al. Myelin sheath structure and regeneration in peripheral nerve injury repair. Proc. Natl Acad. Sci. USA 116, 22347-22352 (2019).

45. Wang, Q. et al. Hyperglycemia exacerbates acetaminophen-induced acute liver injury by promoting liver-resident macrophage proinflammatory response via AMPK/PI3K/AKT-mediated oxidative stress. Cell Death Discov. $\mathbf{5}$ 119 (2019).

46. Aloud, A. A. et al. Galangin, a dietary flavonoid, improves antioxidant status and reduces hyperglycemia-mediated oxidative stress in streptozotocininduced diabetic rats. Redox Rep.: Commun. Free Radic. Res. 22, 290-300 (2017).

47. Li, H. et al. Role of Nrf2 in the antioxidation and oxidative stress induced developmental toxicity of honokiol in zebrafish. Toxicol. Appl. Pharmacol. $\mathbf{3 7 3}$ 48-61 (2019).

48. Tang, Z. et al. Nrf2 drives oxidative stress-induced autophagy in nucleus pulposus cells via a Keap1/Nrf2/p62 feedback loop to protect intervertebral disc from degeneration. Cell Death Dis. 10, 510 (2019).

49. Singh, A. et al. Small molecule inhibitor of NRF2 selectively intervenes therapeutic resistance in KEAP1-deficient NSCLC tumors. ACS Chem. Biol. 11, 3214-3225 (2016)

50. Park, H. T., Kim, J. K. \& Tricaud, N. The conceptual introduction of the "demyelinating Schwann cell" in peripheral demyelinating neuropathies. Glia 67, 571-581 (2019).

51. Okazaki, R. et al. The crucial role of Erk2 in demyelinating inflammation in the central nervous system. J. Neuroinflammation 13, 235 (2016).

52. Gomez-Sanchez, J. A. et al. After nerve injury, lineage tracing shows that Myelin and Remak Schwann cells elongate extensively and branch to form repair Schwann cells, which shorten radically on remyelination. J. Neurosci. 37, 9086-9099 (2017).
53. Fledrich, R. et al. NRG1 type I dependent autoparacrine stimulation of Schwann cells in onion bulbs of peripheral neuropathies. Nat. Commun. 10, 1467 (2019).

54. Salzer, J. L. Schwann cell myelination. Cold Spring Harb. Perspect. Biol. 7, a020529 (2015).

55. Wang, Q. et al. A thermosensitive heparin-poloxamer hydrogel bridges aFGF to treat spinal cord injury. ACS Appl. Mater. interfaces 9, 6725-6745 (2017).

56. Li, J. et al. FGF1 improves functional recovery through inducing PRDX1 to regulate autophagy and anti-ROS after spinal cord injury. J. Cell. Mol. Med. 22, 2727-2738 (2018).

57. Liang, G. et al. Fibroblast growth factor 1 ameliorates diabetic nephropathy by an anti-inflammatory mechanism. Kidney Int. 93, 95-109 (2018).

58. Zhang, C. et al. The prevention of diabetic cardiomyopathy by non-mitogenic acidic fibroblast growth factor is probably mediated by the suppression of oxidative stress and damage. PloS ONE 8, e82287 (2013).

59. Liu, W. et al. Effective treatment of steatosis and steatohepatitis by fibroblast growth factor 1 in mouse models of nonalcoholic fatty liver disease. Proc. Natl Acad. Sci. USA 113, 2288-2293 (2016).

60. Halim, M. \& Halim, A. The effects of inflammation, aging and oxidative stress on the pathogenesis of diabetes mellitus (type 2 diabetes). Diabetes Metab. Syndr. 13, 1165-1172 (2019)

61. Heidari, F. et al. Inflammatory, oxidative stress and anti-oxidative markers in patients with endometrial carcinoma and diabetes. Cytokine 120, 186-190 (2019).

62. Oguntibeju, O. O. Type 2 diabetes mellitus, oxidative stress and inflammation: examining the links. Int. J. Physiol, Pathophysiol. Pharmacol. 11, 45-63 (2019).

63. Kellogg, A. P. \& Pop-Busui, R. Peripheral nerve dysfunction in experimental diabetes is mediated by cyclooxygenase-2 and oxidative stress. Antioxid. Redox Signal. 7, 1521-1529 (2005).

64. Lu, Y. et al. Fibroblast growth factor 21 facilitates peripheral nerve regeneration through suppressing oxidative damage and autophagic cell death. J. Cell. Mol. Med. 23, 497-511 (2019).

65. Kim, M. et al. Heme oxygenase 1 in Schwann cells regulates peripheral nerve degeneration against oxidative stress. ASN Neuro 11, 1759091419838949 (2019).

66. Olagnier, D. et al. Nrf2 negatively regulates STING indicating a link between antiviral sensing and metabolic reprogramming. Nat. Commun. 9, 3506 (2018).

67. Tebay, L. E. et al. Mechanisms of activation of the transcription factor Nrf2 by redox stressors, nutrient cues, and energy status and the pathways through which it attenuates degenerative disease. Free Radic. Biol. Med. 88, 108-146 (2015).

68. Olsson, T., Barcellos, L. F. \& Alfredsson, L. Interactions between genetic, lifestyle and environmental risk factors for multiple sclerosis. Nat. Rev. Neurol. 13, 25-36 (2017).

69. Uruno, A. et al. The Keap1-Nrf2 system prevents onset of diabetes mellitus. Mol. Cell. Biol. 33, 2996-3010 (2013).

70. Jeong, J. Y. et al. Activation of the $\mathrm{Nrf} / \mathrm{HO}-1$ signaling pathway contributes to the protective effects of baicalein against oxidative stress-induced DNA damage and apoptosis in HEl193 Schwann cells. Int. J. Med. Sci. 16, 145-155 (2019).

71. Lin, W. F. et al. SH2B1beta enhances fibroblast growth factor 1 (FGF1)-induced neurite outgrowth through MEK-ERK1/2-STAT3-Egr1 pathway. Cell. Signal. 21, 1060-1072 (2009).

72. Reimers, D., Prieto, R., Gimenez-Gallego, G., Cuevas, P. \& Barrio, L. C. Acidic fibroblast growth factor inhibits junctional communication of Schwann cells in culture. Neurol. Res. 22, 685-691 (2000).

73. Ko, C. C., Tu, T. H., Wu, J. C., Huang, W. C. \& Cheng, H. Acidic fibroblast growth factor in spinal cord injury. Neurospine 16, 728-738 (2019).

74. Lowe, W. L. Jr., Florkiewicz, R. Z., Yorek, M. A., Spanheimer, R. G. \& Albrecht, B. N. Regulation of growth factor mRNA levels in the eyes of diabetic rats. Metab. Clin. Exp. 44, 1038 (1995).

75. Cinci, L. et al. Oxidative, metabolic, and apoptotic responses of Schwann cells to high glucose levels. J. Biochem. Mol. Toxicol. 29, 274-279 (2015).

76. Goncalves, N. P., Vaegter, C. B. \& Pallesen, L. T. Peripheral glial cells in the development of diabetic neuropathy. Front. Neurol. 9, 268 (2018).

77. Gumy, L. F., Bampton, E. T. \& Tolkovsky, A. M. Hyperglycaemia inhibits Schwann cell proliferation and migration and restricts regeneration of axons and Schwann cells from adult murine DRG. Mol. Cell. Neurosci. 37, 298-311 (2008). 